\title{
"Pesquisa e Ensino em Ciências Exatas e da Natureza": um periódico científico multidisciplinar da Unidade Acadêmica de Ciências Exatas e da Natureza (UACEN), Centro de Formação de Professores (CFP), Universidade Federal de Campina Grande (UFCG)
}

\author{
Silvio Felipe B. Lima, Carlos Davidson Pinheiro \& Heydson Henrique B. Silva
}

Universidade Federal de Campina Grande, Centro de Formação de Professores, Unidade Acadêmica de Ciências Exatas e da Natureza, Rua Sérgio Moreira de Figueiredo, S/N, Casas Populares, Cajazeiras 58900000, Paraíba, Brasil. E-mail: sfblima@gmail.com, cdpinheiro@gmail.com, heydson@bol.com.br

Lima S.F.B., Pinheiro C.D. \& Silva H.H.B. (2017) "Pesquisa e Ensino em Ciências Exatas e da Natureza”: um periódico científico multidisciplinar da Unidade Acadêmica de Ciências Exatas e da Natureza (UACEN), Centro de Formação de Professores (CFP), Universidade Federal de Campina Grande (UFCG). Pesquisa e Ensino em Ciências Exatas e da Natureza, 1(1): 01-04.

É com imensa satisfação que apresentamos à comunidade científica o periódico Pesquisa e Ensino em Ciências Exatas e da Natureza (PECEN). O lançamento deste periódico representa um marco importante e de grande significado para a Unidade Acadêmica de Ciências Exatas e da Natureza (UACEN), Centro de Formação de Professores (CFP), Universidade Federal de Campina Grande (UFCG), por consolidar um projeto de longo alcance, tendo em vista a multidisciplinaridade da revista e do corpo editorial envolvido, o qual é composto por docentes da UFCG e de outras instituições de ensino superior do país. A proposta de criação e estruturação do periódico foi iniciada pelo Prof. Dr. Carlos D. Pinheiro (UACEN) no segundo semestre de 2015 e concretizada no primeiro semestre de 2017, com a contratação do Prof. Dr. Silvio F. B. Lima, que passou a gerenciar a implantação e a formatação do periódico, tendo o apoio do Prof. Dr. Heydson H. B. Silva e da equipe editorial apresentada ao final. O nome do periódico foi escolhido para enfatizar o seu caráter multidisciplinar e de divulgação de trabalhos científicos nas mais diversas áreas de Ciências Exatas e da Natureza, principalmente, no que se refere à Física, Matemática, Química e as Ciências Biológicas.

PECEN é um periódico eletrônico, multidisciplinar, semestral, sem custos para a publicação e de livre acesso organizado e editado pelos docentes da UACEN/CFP/UFCG, Campus Cajazeiras, Paraíba. O periódico tem como objetivo publicar contribuições escritas em português ou inglês em todos os campos e aspectos relacionados a pesquisa e ensino em Ciências Exatas e da Natureza. Tais contribuições incluem artigos e notas originais provenientes de pesquisa científica, artigos originais de cunho teórico-metodológico, revisões temáticas da literatura, apresentação de livros, pontos de vista, notícias, opiniões, erratas, obituários e editoriais. A principal missão do periódico é promover a publicação de escritos científicos de qualidade nas diferentes áreas de inserção do periódico, possibilitando assim o desenvolvimento interdisciplinar/multidisciplinar das ciências. Os escritos científicos publicados neste periódico serão divulgados da forma mais ampla possível, principalmente através da busca de indexação em base de dados de relevância científica.

A criação e manutenção de um periódico científico de caráter multidisciplinar não é uma tarefa fácil. Sabendo disto, o corpo docente da UACEN buscou a ajuda de pesquisadores vinculados a outras instituições de ensino superior do país e de comprovada produtividade 
científica nas mais diversas subáreas do conhecimento das Ciências Biológicas, Física, Matemática e Química, para compor o quadro de editores associados. Por outro lado, o caráter multidisciplinar do periódico possibilita a divulgação de pesquisas em um âmbito extremamente amplo dentro da área de conhecimento das Ciências Exatas e da Natureza, o que é algo muito importante, no entanto, incomum entre os periódicos científicos nacionais e internacionais. A equipe editorial do periódico PECEN é formada, em sua maior parte, por doutores que se destacam em suas atividades de ensino e pesquisa em instituições no nordeste, sudeste e sul do país. A qualidade e rigor científico dos trabalhos publicados no periódico representa o resultado da somatória da qualidade de sua equipe editorial.

PEGEN utiliza o Open Journal Systems (OJS) desenvolvido pelo Public Knowledge Project (PKP) (Open Journal Systems-http://pkp.sfu.ca/ojs/), da Universidade British Columbia no Canadá, o qual foi traduzido e customizado pelo Instituto Brasileiro de Informação em Ciência e Tecnologia (IBICT) ((http://www.ibict.br/secao.php?cat=SEER)) recebendo o nome de Sistema Eletrônico de Editoração de Revistas (SEER). Trata-se de um software desenvolvido para a construção e gestão de publicações eletrônicas. Public Knowledge Project é o resultado da experiência de outros sistemas desenvolvidos para preservação de dados (Rosenthal et al. 2005). Dessa forma, esperar-se-á uma grande visibilidade para as publicações do PECEN, a qual será potencializada pelo Protocolo OAI-PMH e outros dispositivos de preservação digital de dados (Batista 2006). Todas as edições estarão disponíveis para acesso irrestrito e sem custos a comunidade científica nacional e internacional, em formato digital "Portable Document Format" (pdf).

Os artigos científicos publicados neste primeiro volume pertencem as mais diferentes subáreas do conhecimento das Ciências Biológicas. O seu conjunto mostra, sobretudo, a importância de um periódico com um escopo multidisciplinar e da divulgação de conhecimentos científicos gerados no país. Tais artigos representam, claramente, o fruto de muito estudo e dedicação de pesquisadores vinculados a instituições distintas do país e também do exterior, demonstrando, de imediato, nesta edição de lançamento, o papel científico de grande relevância do periódico PECEN na divulgação de estudos de qualidade perante a comunidade científica.

No primeiro artigo, João V. Tomotani e Rodrigo B. Salvador analisam o conteúdo de Evolução em livros didáticos do Ensino Fundamental brasileiro. Os autores verificaram que, de modo geral, a Teoria da Evolução não tem sido devidamente apresentada em livros didáticos de séries iniciais havendo problemas capazes de gerar confusão conceitual em estudantes e tópicos relacionados a Evolução de difícil aplicabilidade.

No segundo artigo, Rudá A. Lucena, Silvio F. B. Lima e Martin L. Christoffersen ampliam o conhecimento sobre a riqueza de espécies de Pycnogonida para a costa do estado da Paraíba com base na identificação de Pallenopsis fluminensis. A fauna deste grupo de Chelicerata tem sido muito pouco estudada, ao longo dos anos, na costa Atlântica da América do Sul. Consequentemente, o conhecimento sobre a riqueza de Pycnogonida e inúmeros aspectos biológicos e ecológicos das espécies ainda é extremamente subestimado, principalmente no que tange a fauna da costa brasileira.

No terceiro artigo, Felipe Wartchow apresenta uma revisão da literatura compilando aspectos históricos referentes a Taxonomia, Sistemática e distribuição geográfica dos fungos do gênero Amanita provenientes do Brasil.

No quarto artigo, Jéssica Prata e Martin L. Christoffersen apresentam um checklist das espécies de equinodermos da classe Holothuroidea provenientes da costa do estado da Paraíba. Dezesseis espécies de pepinos-do-mar foram registradas para a costa da região, todas ilustradas com excelente qualidade.

No quinto artigo, Jefferson S. Mikalauskas, Daniel O. Santana e Stephen F. Ferrari documentam, pela primeira vez, o momento em que uma serpente da família Colubridae (popularmente conhecida como falsa-coral) se alimenta de uma espécie de lagarto da família Tropiduridae (popularmente conhecido como calango) ampliando assim o conhecimento sobre as interações ecológicas destes grupos. 
No sexto artigo, Paulo Roberto de Medeiros e Ana Maria Alves de Medeiros estudam a influência de fatores (e.g., rugosidade, exposição às ondas, cobertura do substrato e impactos antrópicos) na distribuição e composição das comunidades de peixes recifais de áreas com um gradiente de restrição a atividades humanas no arquipélago de Fernando de Noronha (nordeste do Brasil). Os resultados deste estudo sugerem que a presença humana, mesmo em áreas fiscalizadas, pode interferir na estrutura dos peixes recifais.

Para finalizar, gostaríamos de agradecer e dedicar este Editorial a todos os autores e todas as autoras que contribuíram com escritos científicos para a edição de lançamento do periódico PECEN.

Boa leitura!

\section{Agradecimentos}

Aos seguintes pesquisadores/pesquisadoras pela importante contribuição na forma de artigos científicos a edição de lançamento do periódico: $\operatorname{Dr}^{a}$ Ana Maria Alves de Medeiros (Universidade Federal da Paraíba, Departamento de Sistemática e Ecologia, Brasil), Dr. Daniel O. Santana (Universidade Federal da Paraíba, Departamento de Sistemática e Ecologia, Brasil), Dr. Felipe Wartchow (Universidade Federal da Paraíba, Departamento de Sistemática e Ecologia, Brasil), M. Sc. Jéssica Prata (Universidade Federal da Paraíba, Departamento de Sistemática e Ecologia, Brasil), M. Sc. Jefferson S. Mikalauskas (Universidade Federal Rural do Rio de Janeiro, Brasil), M. Sc. João Vitor Tomotani (Universidade de São Paulo, Brasil), Dr. Martin Lindsey Christoffersen (Universidade Federal da Paraíba, Departamento de Sistemática e Ecologia, Brasil), Dr. Paulo Roberto de Medeiros (Universidade Federal de Campina Grande, Centro de Formação de Professores, Unidade Acadêmica de Ciência Exatas e da Natureza, Brasil), Dr. Rodrigo Brincalepe Salvador (Staatliches Museum für Naturkunde Stuttgart; Eberhard Karls Universität Tübingen, Alemanha), M. Sc. Rudá Amorim Lucena (Universidade Federal da Paraíba, Departamento de Sistemática e Ecologia, Brasil) e Dr. Stephen F. Ferrari (Universidade Federal de Sergipe, Departamento de Ecologia, Brasil). A todos os consultores Ad Hoc pela revisão crítica dos manuscritos.

\section{Referências}

Batista G.T. (2006) Editorial de lançamento da Revista Ambiente e Água. Ambiente \& Água, 1(1): 3-5.

Rosenthal S.H.D., Robertson R., Reich V. \& Morabito S. (2005) Requirements for digital preservation systems: a bottom-up approach. D-Lib Magazine, 1(11): 1-21. 


\section{EDITORES-CHEFES}

Dr. Carlos Davidson Pinheiro;

M. Sc. Eudes Leite de Lima;

Dr. Heydson Henrique Brito da Silva;

Dr. Silvio Felipe Barbosa de Lima;

\section{COMISSÃO EDITORIAL}

Dra. Albaneide Fernandes Wanderley;

Dr. Carlos Davidson Pinheiro;

M. Sc. Edilson Leite da Silva;

M. Sc. Eudes Leite de Lima;

Dr. Fernando Antônio Portela da Cunha;

M. Sc. Francisco Carlos Pinheiro da Costa;

M. Sc. Gustavo de Alencar Figueiredo;

Dr. Heydson Henrique Brito da Silva;

Dr. João Maria da Silva;

Dra. Mirleide Dantas Lopes;

Dr. Silvio Felipe Barbosa de Lima;

\section{COMISSÃO CIENTÍFICA}

Dra. Albaneide Fernandes Wanderley;

Dr. Carlos Davidson Pinheiro;

M. Sc. Edilson Leite da Silva;

M. Sc. Eudes Leite de Lima;

Dr. Fernando Antônio Portela da Cunha;

M. Sc. Francisco Carlos Pinheiro da Costa;

M. Sc. Gustavo de Alencar Figueiredo;

Dr. Heydson Henrique Brito da Silva;

M. Sc. Hugo da Silva Florentino;

Dr. João Maria da Silva;

Dr. José Deomar de Souza Barros;

Dra. Letícia Carvalho Benitez;

Dra. Mirleide Dantas Lopes;

Dr. Paulo Roberto de Medeiros;

Esp. Rosana Ferreira de Alencar;

Dr. Silvio Felipe Barbosa de Lima;

\section{EDITORES CIENTÍFICOS DE SEÇÃO}

Dra. Albaneide F. Wanderley (Química); Dr. Carlos Davidson Pinheiro (Química); Dr. Fernando A. Portela da Cunha (Química); M. Sc. Edilson Leite da Silva (Computação); M. Sc. Eudes Leite de Lima (Matemática); M. Sc. Franciélia L. de Sousa (Matemática); M. Sc. Gustavo de Alencar Figueiredo (Física); Dr. Heydson Henrique Brito da Silva (Física);
Dra. Mirleide Dantas Lopes (Física);

M. Sc. Francisco Carlos P. Costa (Biologia);

M. Sc. Hugo da Silva Florentino (Biologia);

Dra. Letícia Carvalho Benitez (Biologia);

Dr. Paulo Roberto de Medeiros (Biologia);

Esp. Rosana Ferreira Alencar (Biologia);

Dr. Silvio Felipe Barbosa de Lima (Biologia);

Dr. Udson Santos (Biologia);

\section{EDITORES ASSOCIADOS}

Dra. Antonia Arisdélia A. Feitosa (UFPB/DSE); M. Sc. Arielson Santos Protázio (UFRB/CCAAB); Dr. Carlos Alexandre Borges Garcia (UFS/DQI); Dra. Eugenia Jacira Bolacel Braga (UFPel/IB); Dr. Felipe Wartchow (UFPB/DSE);

Dr. Gilberto Gonçalves Rodrigues (UFPE/CCB); Dr. Hugo Fernandes-Ferreira (UECE/DSE); Dr. Jose Ricardo Miras Mermudes (UFRJ/IB); Dr. Marcelo Fulgêncio Guedes Brito (UFS/DB); Dr. Marcio Bernardino da Silva (UFPB/DSE); Dr. Martin Lindsey Christoffersen (UFPB/DSE); Dr. Mauro de Melo Júnior (UFRPE/DB); Dra. Michela Borges (UNICAMP/IB/MZ); Dr. Paulo Henrique R. Peixoto (UFPE/CAA); Dra. Rita de Cássia M. C. Rodriguez (UFPel/IB); Dr. Rodrigo Giesta Figueiredo (UFES/CCENS); Dr. Samuel Vieira Brito (UFMA/CCAA); Dr. Sérgio André Fontes Azevedo (UFPB/DF); Dra. Thatyara Freire de Souza (UFERSA/CMPF); Dr. Wedson de Medeiros S. Souto (UFPI/CCN);

\section{EDITORES ASSISTENTES E DE PRODUÇÃO}

Sra. Ariel Rodrigues Holanda; Sra. Bianca Vieira Lima;

M. Sc. Eudes Leite de Lima;

Dr. Heydson Henrique Brito da Silva; Sra. Moniky Mendes Maciel; Dr. Onireves Monteiro de Castro (UFCG); Sra. Pricila Bento Gonçalves; Sr. Renato de Freitas Souza; Dr. Silvio Felipe Barbosa de Lima; Sra. Talyta Karoline Santos Oliveira;

\section{APOIO TÉCNICO OPERACIONAL E PRODUÇÃO GRÁFICA}

Sr. Antonio Lourenço de Araújo Filho; Sr. Délio Jackson Dantas Duarte; 